\title{
LOWER BOUND TO A PROBLEM OF MOCANU ON DIFFERENTIAL SUBORDINATION
}

\author{
S. PONNUSAMY
}

\begin{abstract}
Let $\mathcal{S}^{*}$ denote the family of starlike mappings in the unit disc $\Delta$. Let $\mathcal{R}(\alpha, \beta)$ denote the family of normalized analytic functions in $\Delta$ satisfying the condition $\operatorname{Re}\left(f^{\prime}(z)+\alpha z f^{\prime \prime}(z)\right)>\beta, z \in \Delta$ for some $\alpha>0$. In this note, among other things, we give a lower bound to the problem of Mocanu aimed at determining $\inf \left\{\alpha: R(\alpha, 0) \subset \mathcal{S}^{*}\right\}$.
\end{abstract}

\section{Introduction and Preliminaries}

We denote the unit disc in the complex plane, that is $\{z \in \mathbb{C}:|z|<1\}$, by $\Delta$. The family $\mathcal{A}$ is the family of all functions $f$ which are analytic in the unit disc $\Delta$ and which satisfiy $f(0)=f^{\prime}(0)-1=0$. Let us denote by $\mathcal{S}^{*}, \mathcal{C}$ and $\mathcal{S}$ the well-known sub families of $\mathcal{A}$ consisting of starlike, close-to-convex and univalent functions respectively. A sufficient condition for $f \in \mathcal{A}$ to be in $\mathcal{C}$ is that $\operatorname{Re} f^{\prime}(z)>0$ in $\Delta$. In 1986 Mocanu [3] proved the following:

$$
\mathcal{R}(\alpha, 0) \subset \mathcal{S}^{*} \text { at least when } \alpha \geq 1 / 2
$$

where

$$
\mathcal{R}(\alpha, \beta)=\left\{f \in \mathcal{A}: \operatorname{Re}\left(f^{\prime}(z)+\alpha z f^{\prime \prime}(z)\right)>\beta, z \in \Delta\right\} .
$$

Further, in the same article Mocanu raised the problem of determining

$$
\inf \left\{\alpha: \mathcal{R}(\alpha, 0) \subset \mathcal{S}^{*}\right\}
$$

However, in [4] the author improved the result (1.1) in the following way:

$$
\mathcal{R}\left(\alpha, \beta\left(0, \alpha, \alpha^{\prime}\right)\right) \subset \mathcal{S}^{*} \quad \text { at least when } \alpha \geq \alpha^{\prime} \approx 0.426
$$

Received November 10, 1993; revised June 1, 1995.

1991 Mathematics Subject Classification. 30C45.

Key words and phrases. Subordination, starlike and univalent functions. 
where

$$
\beta\left(\delta, \alpha, \alpha^{\prime}\right)=\frac{\delta-\left(1-\alpha^{\prime} / \alpha\right)(2 \rho(\alpha)-1)}{1-\left(1-\alpha^{\prime} / \alpha\right)(2 \rho(\alpha)-1)}
$$

and

$$
\rho(\alpha)=\int_{0}^{1} \frac{d t}{1+t^{\alpha}} .
$$

The purpose of this note is to determine a lower bound $\alpha^{\prime}$ to the problem of Mocanu such that

$$
\mathcal{R}\left(\alpha, \beta\left(0, \alpha, \alpha^{\prime}\right)\right) \subset \mathcal{S}^{*} \text { for } \alpha \geq \alpha^{\prime} \approx 0.4036 .
$$

Connected to this a ralated but general result is also derived.

For the proof of our result we need the following lemmas:

Lemma 1.5.([5]) Suppose that $\alpha \geq \alpha^{\prime}>0, \delta<1$ and $\beta\left(\delta, \alpha, \alpha^{\prime}\right)$ is defined by (1.3). Then

$$
\mathcal{R}\left(\alpha, \beta\left(\delta, \alpha, \alpha^{\prime}\right)\right) \subset \mathcal{R}\left(\alpha^{\prime}, \delta\right)
$$

Lemma 1.6. ([6, Lemma 1]) Let $\beta_{0}$ be the solution of

$$
\beta \pi=\frac{3 \pi}{2}-\arctan \eta
$$

for a suitable fixed $\eta>0$ so that $\lambda(z): \Delta \longrightarrow \mathbb{C}$ satisfies

$$
|\operatorname{Im} \lambda(z)| \leq \frac{1}{\eta}\left(\operatorname{Re} \lambda(z)-\frac{\eta}{\beta}\right), z \in \Delta
$$

and let

$$
\alpha=\alpha(\beta, \eta)=\beta+\frac{2}{\pi} \arctan \eta, \quad 0<\beta \leq \beta_{0} .
$$

If $p$ is analytic in $\Delta$ with $p(0)=1$ and satisfies

$$
\left|\arg \left(p(z)+\lambda(z) z p^{\prime}(z)\right)\right|<\frac{\pi}{2} \alpha, z \in \Delta
$$

then

$$
|\arg p(z)|<\frac{\pi}{2} \beta, \quad z \in \Delta
$$

Lemma 1.7. ([1,2,3]) Let $\Omega$ be a set in the complex plane $\mathbb{C}$ and suppose that $\psi: \mathbb{C}^{2} \times \Delta \longrightarrow \mathbb{C}$ satisfies the condition

$$
\psi(i x, y ; z) \notin \Omega, \text { for } z \in \Delta \text { and real } x, y \text { with } y \leq-\left(1+x^{2}\right) / 2 \text {. }
$$

If $p$ is analytic in $\Delta, p(0)=1$, and if $\psi\left(p(z), z p^{\prime}(z) ; z\right) \in \Omega$ then $\operatorname{Re} p(z)>0$ for $z \in \Delta$. 
All of the inequalities in this article involving functions of $z$ hold uniformly in the unit disc $\Delta$. Therefore, the condition ' $z \in \Delta$ ' will be omitted in the remainder of the article.

\section{Lower Bound to the Problem of Mocanu}

Lemma 1.6 has found a number of interesting applications in [5]. Apart from these, a very important and useful special case yields the following theorem.

Theorem 2.1. If $p$ is analytic in $\Delta$, with $p(0)=1$, and satisfies

$$
\operatorname{Re}\left(p(z)+\alpha z p^{\prime}(z)\right)>-\frac{\left(1-\alpha^{\prime} / \alpha\right)(2 \rho(\alpha)-1)}{1-\left(1-\alpha^{\prime} / \alpha\right)(2 \rho(\alpha)-1)}
$$

for some $\alpha \geq \alpha^{\prime} \equiv 3 \cot ((1+\varepsilon) \pi / 3) /(2(1+\varepsilon)),-1<\varepsilon \leq 1 / 2$, then

$$
|\arg p(z)|<\frac{(1+\varepsilon) \pi}{3}
$$

where $\rho(\alpha)$ is defined by (1.4).

Proof. In view of Lemma 1.5, with $p(z)=f^{\prime}(z)$, and (2.2) it suffices to show that

$$
\operatorname{Re}\left(p(z)+\alpha^{\prime} z p^{\prime}(z)\right)>0 \text { implies }|\arg p(z)|<\frac{(1+\varepsilon) \pi}{3} .
$$

From the condition on $\alpha^{\prime}$ and $\varepsilon$, it is easy to see that

$$
1=\frac{2(1+\varepsilon)}{3}+\frac{2}{\pi} \arctan \left(\frac{2 \alpha^{\prime}(1+\varepsilon)}{3}\right) .
$$

In Lemma 1.6, we choose $\lambda(z) \equiv \alpha^{\prime}, \alpha=1, \beta=2(1+\varepsilon) / 3$ and $\eta=\alpha^{\prime} \beta$. Then (2.3) follows from Lemma 1.6 and therefore the proof of the theorem is complete.

For $\lambda(z)=1$ and $\eta=\beta$ in Lemma 1.6 the following simple observation will be useful (see also [2]): if $f \in \mathcal{A}$ then

$$
\left|\arg f^{\prime}(z)\right|<\frac{(1+\varepsilon) \pi}{3} \text { implies }\left|\arg \frac{f(z)}{z}\right|<\frac{\pi \beta}{2},
$$

where $0<\beta \leq \beta_{0}$ is given by

$$
\frac{2(1+\varepsilon)}{3}=\beta+\frac{2}{\pi} \arctan \beta,(-1<\varepsilon \leq 2)
$$

and $\beta_{0}$ is the solution of

$$
y \pi=\frac{3 \pi}{2}-\arctan y
$$


In particular, we have

$$
\text { Re } f^{\prime}(z)>0 \text { implies }\left|\arg \frac{f(z)}{z}\right|<\frac{\pi \beta}{2},
$$

where $\beta \approx 0.63$ in $(2.5)$ is the solution of $x=\cot (x \pi / 2)$.

Example 2.6. From Lemma 1.6, it is simple to show that if $f \in \mathcal{A}$ satisfies

$$
\operatorname{Re}\left(f^{\prime}(z)+(\sqrt{3} / 2) z f^{\prime \prime}(z)\right)>0
$$

then we have

$$
|\arg f(z)|<\frac{\pi}{3} .
$$

This suggests that there may exist a $\delta>0$ and an $\varepsilon$ with $1 / 3<\varepsilon \leq 1 / 2$ for which

$$
\operatorname{Re}\left(f^{\prime}(z)+\alpha z f^{\prime \prime}(z)\right)>-\delta \text { implies }\left|\arg f^{\prime}(z)\right|<\varepsilon \pi,
$$

for some $\alpha<\sqrt{3} / 2$. A general solution to this problem will be presented later.

Choose $\alpha=1 / 2$ in Theorem 2.1. Therefore, for $1 / 3<\varepsilon \leq 1 / 2$ satisfying the condition that $\varepsilon \tan (\varepsilon \pi) \geq 1$, we have

$$
\operatorname{Re}\left(f^{\prime}(z)+\frac{1}{2} z f^{\prime \prime}(z)\right)>-\frac{[1-1 /(\varepsilon \tan (\varepsilon \pi))][3-4 \log 2]}{1-[1-1 /(\varepsilon \tan (\varepsilon \pi))][3-4 \log 2]}
$$

which implies

$$
\left|\arg f^{\prime}(z)\right|<\varepsilon \pi
$$

Theorem 2.7. Let $\varepsilon \approx 0.3059$ be the solution of

$$
\frac{(1+\varepsilon) \pi}{3}=\beta+\arctan \left(\frac{2 \beta}{\pi}\right)
$$

with

$$
\beta=\arctan \left(\frac{\sqrt{3} \alpha^{\prime}}{1-\alpha^{\prime}}\right)
$$

and

$$
\alpha^{\prime}=\frac{3 \cot ((1+\varepsilon) \pi / 3)}{2(1+\varepsilon)} .
$$

If $f \in \mathcal{A}$ satisfies

$$
\operatorname{Re}\left(f^{\prime}(z)+\alpha z f^{\prime \prime}(z)\right)>-\frac{\left(1-\alpha^{\prime} / \alpha\right)(2 \rho(\alpha)-1)}{1-\left(1-\alpha^{\prime} / \alpha\right)(2 \rho(\alpha)-1)}
$$

for $\alpha \geq \alpha^{\prime} \approx 0.4036$, then $f \in \mathcal{S}^{*}$. 
Proof. By Lemma 1.5, it is sufficient to prove the theorem for $\alpha=\alpha^{\prime}$. Suppose that $f$ satisfies

$$
\operatorname{Re}\left(f^{\prime}(z)+\alpha^{\prime} z f^{\prime \prime}(z)\right)>0
$$

Then, by Theorem 2.1, we obtain

$$
\left|\arg f^{\prime}(z)\right|<\frac{(1+\varepsilon) \pi}{3}
$$

which, by (2.4), implies that

$$
\left|\arg \frac{f(z)}{z}\right|<\arctan \left(\frac{\sqrt{3} \alpha^{\prime}}{1-\alpha^{\prime}}\right) .
$$

with

$$
\frac{(1+\varepsilon) \pi}{3}=\arctan \left(\frac{\sqrt{3} \alpha^{\prime}}{1-\alpha^{\prime}}\right)+\arctan \left\{\frac{2}{\pi} \arctan \left(\frac{\sqrt{3} \alpha^{\prime}}{1-\alpha^{\prime}}\right)\right\},
$$

which is in fact equivalent to (2.8).

Now, if we let $p(z)=z f^{\prime}(z) / f(z)$ then $p$ is analytic in $\Delta$ with $p(0)=1$. Further, by arithmetic calculations, (2.9) can be written as

$$
\psi\left(p(z), z p^{\prime}(z) ; z\right) \in \Omega=\{\omega \in \mathbb{C}: \operatorname{Re} \omega>0\}
$$

where

$$
\psi(r, s ; z)=\alpha^{\prime} \frac{f(z)}{z}\left[r^{2}+\left(\frac{1}{\alpha^{\prime}}-1\right) r+s\right] .
$$

We wish to show that $\operatorname{Re} p(z)>0$ in $\Delta$ which is equivalent to $f \in \mathcal{S}^{*}$. If we let $f(z) / z=U+i V$ then we can easily find that

$$
\operatorname{Re} \psi(i x, y ; z)=\alpha^{\prime}\left[\left(y-x^{2}\right) U-\left(1 / \alpha^{\prime}-1\right) V x\right]
$$

and, therefore, we deduce that

$$
\operatorname{Re} \psi(i x, y ; z) \leq-\frac{\alpha^{\prime}}{2}\left[3 U x^{2}+2\left(\frac{1}{\alpha^{\prime}}-1\right) V x+U\right] \equiv Q(x)
$$

for $y \leq-\left(1+x^{2}\right) / 2$ and $x$ real. Since $f(z) / z=U+i V$, we may rewrite (2.10) as

$$
|V|<\left(\frac{\sqrt{3} \alpha^{\prime}}{1-\alpha^{\prime}}\right) U
$$

and so the discriminant of $Q(x)$ is non-positive. This implies that $\operatorname{Re} \psi(i x, y ; z) \leq 0$ for $z \in \Delta, x$ real and $y \leq-\left(1+x^{2}\right) / 2$. Hence, by Lemma 1.7 and Equation (1.8), we conclude that

$$
\operatorname{Re} p(z)=\operatorname{Re}\left(z f^{\prime}(z) / f(z)\right)>0
$$


and, therefore, $f$ is starlike. This completes the proof of the Theorem 2.7.

As an immediate application of Lemma 1.6 we have the following:

Corollary 2.12. If $\lambda$ is a function defined on $\Delta$ such that

$$
|\operatorname{Im} \lambda(z)| \leq \tan \left(\frac{\pi \gamma}{2}\right)\left[\operatorname{Re} \lambda(z)-\frac{\cot (\pi \gamma / 2)}{\gamma}\right]
$$

for $0<\gamma<1$, then for $p$ analytic in $\Delta$ with $p(0)=1$

$$
\operatorname{Re}\left(p(z)+\lambda(z) z p^{\prime}(z)\right)>0 \text { implies }|\arg p(z)|<\frac{\pi \gamma}{2} .
$$

For our next result we generalize Corollary 2.12 as follows:

Theorem 2.13. Let $\lambda$ be a function defined on $\Delta$ satisfying

$$
|\operatorname{Im} \lambda(z)|<\tan \left(\frac{\pi \gamma}{2}\right)[\operatorname{Re} \lambda(z)-\eta],(\eta>0)
$$

for $0<\gamma<1$. If $p$ is analytic in $\Delta$ with $p(0)=1$ and

$$
\operatorname{Re}\left(p(z)+\lambda(z) z p^{\prime}(z)\right)>\beta(\gamma, \eta)
$$

where

$$
\beta(\gamma, \eta)=t_{0}^{\gamma} \cos \left(\frac{\pi \gamma}{2}\right)\left[1-\frac{\gamma \eta}{2}\left(t_{0}+\frac{1}{t_{0}}\right) \tan \left(\frac{\pi \gamma}{2}\right)\right]
$$

with

$$
t_{0}=\frac{1}{\eta(1+\gamma)}\left[\cot \left(\frac{\pi \gamma}{2}\right)\left\{1+\sqrt{1+\eta^{2}\left(1-\gamma^{2}\right) \tan ^{2}(\pi \gamma / 2)}\right\}\right]
$$

then

$$
|\arg p(z)|<\frac{\pi \gamma}{2}
$$

The following corollary is an elementary consequence of the above theorem and so we omit it derivation.

Corollary 2.18. Let $\alpha$ be a complex number satisfying the condition

$$
\operatorname{Re} \alpha>|\operatorname{Im} \alpha| \cot (\pi \gamma / 2) \text { with } 0<\gamma<1 \text {. }
$$

If $p$ is analytic in $\Delta, p(0)=1$ and

$$
\operatorname{Re}\left(p(z)+\alpha z p^{\prime}(z)\right)>\beta(\gamma, \operatorname{Re} \alpha-|\operatorname{Im} \alpha| \cot (\pi \gamma / 2)),
$$


where $\beta$ is as in Theorem 2.13, then

$$
|\arg p(z)|<\frac{\pi \gamma}{2}
$$

Remark 2.19. It can be easily shown that $\beta(\gamma, \eta)=0$ when $\eta=[\cot (\pi \gamma / 2)] / \gamma$. Therefore, Theorem 2.13 is an extension of Corollary 2.12. The case $\gamma=2 / 3$ has already been considered by the author in a different context and further, this particular case has been used to obtain a new sufficient condition for a function $f \in \mathcal{A}$ to be starlike in $\Delta$; see [6] for details. However, it will be interesting if one can use Corollary 2.18 (with $\left.p(z)=f^{\prime}(z)\right)$ and the method of proof used in Theorem 2.7 to determine the correct range of the complex constant $\alpha$ for which $\mathcal{R}(\alpha, 0) \subset \mathcal{S}^{*}$. In [4] the author determined simple conditions on $\alpha \in \mathbb{C}$ and $\beta<1$ for which

$$
f \in \mathcal{A} \text { and } \operatorname{Re}\left(f^{\prime}(z)+\alpha z f^{\prime \prime}(z)\right)>\beta \text { implies } f \in \mathcal{S}^{*} .
$$

Remark 2.20. Let $M$ and $N$ be analytic in $\Delta$ with $M(0)=N(0)=0$, and $M^{\prime}(0) / N^{\prime}(0)=1$. Further, suppose that $N$ maps $\Delta$ onto a multisheeted domain with respect to the origin. Now we consider

$$
\lambda(z)=\frac{\alpha N(z)}{z N^{\prime}(z)} \text { and } p(z)=\frac{M(z)}{N(z)}(\alpha>0) .
$$

Then $\lambda$ and $p$ are analytic in $\Delta, \operatorname{Re} \lambda(z)>0$ in $\Delta, p(0)=1, \lambda(0)=\alpha$ and

$$
p(z)+\lambda(z) z p^{\prime}(z)=(1-\alpha) \frac{M(z)}{N(z)}+\alpha \frac{M^{\prime}(z)}{N^{\prime}(z)} .
$$

If we allow $\gamma \rightarrow 1^{-}$in Corollary 2.12, then, because of the above observation, we easily obtain

$$
\operatorname{Re}\left((1-\alpha) \frac{M(z)}{N(z)}+\alpha \frac{M^{\prime}(z)}{N^{\prime}(z)}\right)>0 \text { implies } \operatorname{Re}\left(\frac{M(z)}{N(z)}\right)>0 .
$$

This is a well-known result of Libera-Sakaguchi-Chichra (see for example [4]). This particular result has a number of applications and hence we expect that Theorem 2.13 may prove to be a useful tool in obtaining new results as well as in improving many of the known results available in the literature.

Proof of Theorem 2.13. Let us write

$$
q(z)=\left(\frac{1+z}{1-z}\right)^{\gamma}, 0<\gamma<1
$$

Then we see that the function $q$ is convex in $\Delta$ and $q(0)=1$. For convenience, we set

$$
H(z)=p(z)+\lambda(z) z p^{\prime}(z)
$$


Now we will use a well-known result of Miller and Mocanu [2] (For a more general form we refer to [1]). Suppose that $p$ is not subordinate to $q$. According to Miller and Mocanu's result, there are points $z_{0} \in \Delta$ and $\zeta_{0} \in \partial \Delta$, and $m \geq 1$ such that

$$
H\left(z_{0}\right)=p\left(z_{0}\right)+\lambda\left(z_{0}\right) z_{0} p^{\prime}\left(z_{0}\right)=q\left(\zeta_{0}\right)+m \lambda\left(z_{0}\right) \zeta_{0} q^{\prime}\left(\zeta_{0}\right)
$$

If we let $\lambda\left(z_{0}\right)=U+i V\left(\equiv \operatorname{Re} \lambda\left(z_{0}\right)+i \operatorname{Im} \lambda\left(z_{0}\right)\right)$, then from (2.14) we, in particular, find that

$$
\left.\begin{array}{l}
U+V \cot (\pi \gamma / 2) \\
U-V \cot (\pi \gamma / 2)
\end{array}\right\} \geq U-|V| \cot (\pi \gamma / 2) \geq \eta>0 .
$$

Using (2.23), (2.22) becomes

$$
H\left(z_{0}\right)=q\left(\zeta_{0}\right)+m(U+i V) \zeta_{0} q^{\prime}\left(\zeta_{0}\right)
$$

Now we need to consider separately the case $p\left(z_{0}\right) \neq 0$ which corresponds to a point on one of the rays on the sector $q(\Delta)$, and the case $p\left(z_{0}\right)=0$ which corresponds to the corner of the sector. Observe that the latter case occurs only when $\gamma \geq 1$. Since $0<\gamma<1$, it is sufficient to consider the case $p\left(z_{0}\right) \neq 0$. In this case we note that $\zeta_{0} \neq \pm 1$. Therefore, if we let $i x=\left(1+\zeta_{0}\right) /\left(1-\zeta_{0}\right)$ and use (2.22) and (2.24), we obtain that

$$
H\left(z_{0}\right)=(i x)^{\gamma}\left[1+i m \gamma(U+i V)\left(\frac{1+x^{2}}{2 x}\right)\right],
$$

where

$$
i x= \begin{cases}x e^{i \pi / 2} & \text { if } x>0 \\ -x e^{-i \pi / 2} & \text { if } x<0\end{cases}
$$

Therefore, we haye

$$
\operatorname{Re} H\left(z_{0}\right)= \begin{cases}|x|^{\gamma} \cos \left(\frac{\pi \gamma}{2}\right)\left[1-\frac{m \gamma}{2}\left(|x|^{-1}+|x|\right)\left(U \tan \left(\frac{\pi \gamma}{2}\right)+V\right)\right] & \text { if } x>0 \\ |x|^{\gamma} \cos \left(\frac{\pi \gamma}{2}\right)\left[1-\frac{m^{2} \gamma}{2}\left(|x|^{-1}+|x|\right)\left(U \tan \left(\frac{\pi \gamma}{2}\right)-V\right)\right] & \text { if } x<0\end{cases}
$$

Thus, from (2.23) and the fact that $\eta>0$ and $m \geq 1$, the above identity reduces to

$$
\operatorname{Re} H\left(z_{0}\right) \leq|x|^{\gamma} \cos \left(\frac{\pi \gamma}{2}\right)\left[1-\frac{\eta \gamma}{2}\left(|x|^{-1}+|x|\right) \tan \left(\frac{\pi \gamma}{2}\right)\right] \equiv g(|x|), \text { say, }
$$

for $x \neq 0$, where

$$
g(t)=t^{\gamma} \cos \left(\frac{\pi \gamma}{2}\right)\left[1-\frac{\eta \gamma}{2}\left(t^{-1}+t\right) \tan \left(\frac{\pi \gamma}{2}\right)\right], \quad t=|x|>0 .
$$

Since

$$
t_{0}=\frac{1}{\eta(1+\gamma)}\left[\cot \left(\frac{\pi \gamma}{2}\right)\left\{1+\sqrt{1+\eta^{2}\left(1-\gamma^{2}\right) \tan ^{2}(\pi \gamma / 2)}\right\}\right]
$$


is the point of maximum for $g(t)$, it follows that for $z_{0}$ in $\Delta$,

$$
\operatorname{Re} H\left(z_{0}\right) \leq g(|x|) \leq g\left(t_{0}\right) \equiv \beta(\gamma, \eta)
$$

where $\beta(\gamma, \eta)$ is defined by (2.16). The above inequality contradicts (2.15) at $z=z_{0} \in \Delta$ and hence we conclude that $p(z)$ is subordinate to $q(z)=((1+z) /(1-z))^{\gamma}$ with $0<\gamma<1$.

We conclude with the following remark: This paper is a part of an internal report of School of Mathematics, SPIC Science Foundation from March 91 and the work was supported by NBHM. After the revised version of the paper was sent for publication, the author learnt that the inclusion $\mathcal{R}(\alpha, \beta(0, \alpha, 1 / 3)) \subset \mathcal{S}^{*}$ holds for $\alpha \geq 1 / 3$ and is an improvement of Theorem 2.7. This result has been obtained in a recent paper by $R$. Fournier and St. Ruscheweyh [On two extremal problems related to univalent functions, Rocky Mountain J. Math. 24(2)(1994), 529-538] and is seem to be the better lower bound for the problem of Mocanu.

\section{References}

[1] S. S. Miller and P. T. Mocanu, "Second order differential inequalities in the complex plane," $J$. Math. Anal. Appl., 65(1978), 289-305.

[2] S. S.Miller and P. T. Mocanu, "Marx-Strohhäcker differential subordinations systems," Proc. Amer. Math. Soc., 99(1987), 527-534.

[3] P. T. Mocanu, "On starlikeness of Libera transform," Mathematica (Cluj), 28(51)(1986), 153-155.

[4] S. Ponnusamy, "Differential subordination and starlike functions," Complex variables: Theory and Appln., 19 (1992), 185-194.

[5] S. Ponnusamy, "Differential subordination concerning starlike functions," Internal Report, SPIC Science Foundation, 1990; (Also Proc. Indian Acad. Sci. (Math. Sci.), 104(1994), 397-411).

[6] S. Ponnusamy, "Differential subordination and Bazilevič function," Internal Report, SPIC Science Foundation, 1990; (Also Proc. Indian Acad. Sci. (Math. Sci.), (1995))

Department of Mathematics, University of Helsinki, P.O. Box 4 (Hallituskatu 15), FIN-00014, University of Helsinki, Finland.

E-mail: samy@geom.helsinki.fi 